\title{
Electrical Spin Injection into High Mobility 2D Systems
}

\author{
M. Oltscher, M. Ciorga, M. Utz, D. Schuh, D. Bougeard, and D. Weiss \\ Experimentelle und Angewandte Physik, University of Regensburg, D-93040 Regensburg, Germany
}

(Received 14 March 2014; published 4 December 2014)

\begin{abstract}
We report on spin injection into a high mobility 2D electron system confined at an ( $\mathrm{Al}, \mathrm{Ga}) \mathrm{As} / \mathrm{GaAs}$ interface, using (Ga,Mn)As Esaki diode contacts as spin aligners. We measured a clear nonlocal spin valve signal, which varies nonmonotonically with the applied bias voltage. The magnitude of the signal cannot be described by the standard spin drift-diffusion model, because at maximum this would require the spin polarization of the injected current to be much larger than $100 \%$, which is unphysical. A strong correlation of the spin signal with contact width and electron mean free path suggests that ballistic transport in the $2 \mathrm{D}$ region below ferromagnetic contacts should be taken into account to fully describe the results.
\end{abstract}

DOI: 10.1103/PhysRevLett.113.236602

PACS numbers: 72.25.Dc, 72.25.Hg, 72.25.Mk, 73.40.Kp

All-electrical spin injection and detection, one of the key ingredients for functional spintronics devices, has been successfully realized in bulk semiconductors like GaAs $[1,2]$ and $\mathrm{Si}[3,4]$. To implement the spin-transistor functionality, proposed by Datta and Das [5], one needs the capacity to controllably rotate the spin of electrons on the way from source to drain. It has been suggested [5] to employ Rashba spin-orbit interaction, due to which the $k$ vector of an electron in a two-dimensional electron gas (2DEG) is connected to an effective magnetic field, which is in turn tunable by an electric field. Thus, the precession angle in a sufficiently narrow channel can be coherently controlled by a gate voltage, provided transport between source and drain is ballistic. Hence, high mobility 2D systems are needed for these experiments. Although spin injection into graphene, a truly 2D system, has already been realized [6,7], no experiments exist in the ballistic regime where the injector or detector widths or their separation is significantly smaller than the electron mean free path $l_{\mathrm{mf}}$. Spin injection into semiconductor-based 2DEGs turns out to be difficult and only a few reports are available [8-11]. The analysis of experiments is also complicated by the lack of a corresponding ballistic theory. Although different aspects of ballistic spin injection and spin transport have been discussed theoretically [12-17], there is no comprehensive theory that would allow us to describe the experimental outcome in this regime in a way in which the spin drift-diffusion theory describes experiments on devices with bulk 3D channels $[1,2]$.

In this Letter we provide clear evidence of spin injection into a high mobility 2DEG confined in an inverted $(\mathrm{Al}, \mathrm{Ga}) \mathrm{As} / \mathrm{GaAs}$ heterojunction, employing the ferromagnetic (FM) semiconductor (Ga,Mn)As in an Esaki diode configuration [18-21] as a spin aligner. We observed a dramatically enhanced nonlocal spin valve (NLSV) signal at negatively biased Esaki junctions when electrons can tunnel directly from (Ga,Mn)As into 2DEG states. At maximum the amplitude of the signal is much larger than predicted by the standard spin drift-diffusion model. The signal is especially large when the contact width is much smaller than the electron mean free path $l_{\mathrm{mf}}$ of the transport channel and scales, as a function of temperature, with $l_{\mathrm{mf}}$. Therefore, we assume that ballistic size effects contribute to the observed enhancement.

The layout of a typical device is sketched in Fig. 1(a). It consists of a $w=50-\mu \mathrm{m}$-wide and 750- $\mu \mathrm{m}$-long lateral channel oriented along $y \|[110]$, with six FM Esaki diode contacts above the nonmagnetic (NM) channel. The center contacts are $0.5 \mu \mathrm{m}$ (contact 2) and $4 \mu \mathrm{m}$ (contacts 3-5) wide. The center-to-center spacing between contacts is $4.25 \mu \mathrm{m}$ for the contact pair $2-3$ and $6 \mu \mathrm{m}$ for pairs $3-4$ and 4-5. Each contact can be used either as an injector or detector. In Fig. 1(a) the nonlocal (NL) setup used for most of the experiments described below is shown, with the narrow contact 2 as the injector and the three wide contacts (3-5) as detectors. According to the drift-diffusion model, spin accumulation is generated in the channel by a charge current flowing through the FM-NM interface along the $z$ direction. This accumulation diffuses away from the injector ( $y$ direction) and gets detected by another FM contact, via spin-charge coupling [22]. Two $150 \mu \mathrm{m} \times 150 \mu \mathrm{m}$ large contacts $(1,6)$ are used as reference. The three terminal voltage $V_{3 T}$ measures the voltage drop across the interface and is also used to monitor the magnetic behavior of the contact [23].

Spin injection requires that (i) a high-quality $2 \mathrm{D}$ channel is formed at the interface, (ii) the charge current, flowing through the contact to the channel, is large enough to generate a sizable spin accumulation in the 2DEG, and (iii) lateral transport occurs exclusively within the 2D layer. To achieve that, we employed the Nextnano simulator [24] to find optimal parameters for our devices. These were fabricated from the wafer sketched in Fig. 1(b). It was grown by MBE on a semi-insulating (001) GaAs substrate and consists of the following layers (order of growth): $1000 \mathrm{~nm} \mathrm{GaAs} /(\mathrm{Al}, \mathrm{Ga})$ As superlattice (not shown), 


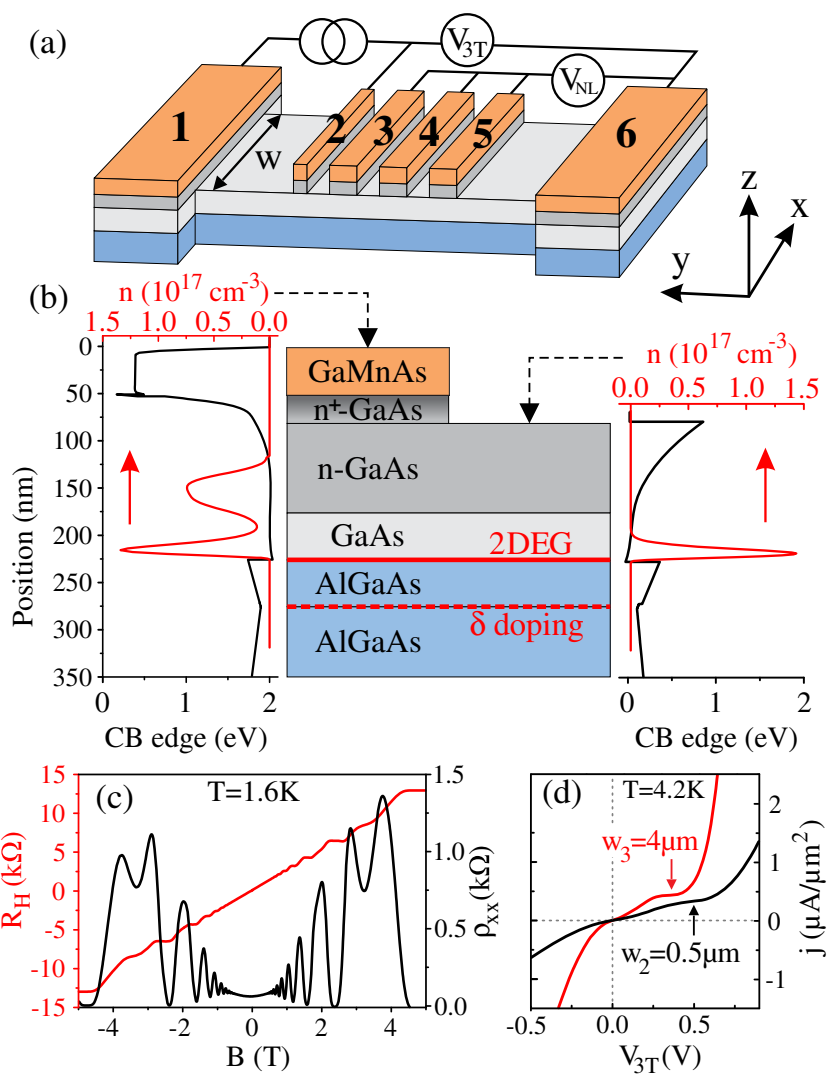

FIG. 1 (color online). (a) Sample layout and the main measurement configuration described in the text. (b) The layer sequence of the device; on the left- and right-hand sides we show simulated conduction band edge profiles (black lines) together with the electrons' density (red lines) at $T=4.2 \mathrm{~K}$ for a complete layer stack and with the highly doped top layers etched away, respectively. (c) Magnetotransport measurements on a standard Hall bar device. (d) $I-V$ characteristics of contacts 2 and 3 taken in the three-terminal geometry. The arrows mark positions of "the Esaki kink" for the each curve.

$75 \mathrm{~nm} \mathrm{Al}_{0.33} \mathrm{Ga}_{0.67} \mathrm{As}$ with a Si $\delta$-doping layer, $50 \mathrm{~nm}$ undoped $\mathrm{Al}_{0.33} \mathrm{Ga}_{0.67} \mathrm{As}$ buffer layer, $50 \mathrm{~nm}$ undoped GaAs, $100 \mathrm{~nm}$ lightly Si-doped GaAs with doping density $n=7 \times 10^{16} \mathrm{~cm}^{-3}, 15 \mathrm{~nm} n \rightarrow n^{+}$transition layer with $n^{+}=5 \times 10^{18} \mathrm{~cm}^{-3}$, and a $2.2 \mathrm{~nm} \mathrm{Al}_{0.33} \mathrm{Ga}_{0.67}$ As layer. The top layer of $50 \mathrm{~nm}(\mathrm{Ga}, \mathrm{Mn}) \mathrm{As}$ has been grown using low-temperature MBE. This layer and the $n^{+}$-GaAs layers form an Esaki diode; the 2DEG is confined at the $(\mathrm{Al}, \mathrm{Ga}) \mathrm{As} / \mathrm{GaAs}$ interface. Contacts were defined by optical or $e$-beam lithography and subsequent evaporation of Ti/Au. Finally, the highly doped top layers are etched away between the contacts to limit the lateral transport to the $2 \mathrm{D}$ system.

In Fig. 1(b), left, we show the corresponding Nextnano band-profile simulation. In addition to the 2DEG confined at the $(\mathrm{Al}, \mathrm{Ga}) \mathrm{As} / \mathrm{GaAs}$ interface, there exists a region of finite electron concentration within the mildly doped $n$-GaAs layer. This $n$-doped bulk region enables efficient charge transport between (Ga,Mn)As and the 2DEG. To avoid lateral transport parallel to the 2DEG, mobile carriers in the doped GaAs layers were depleted between the contacts by etching the top layers away. The etching depth $d$ is crucial: if $d$ is too large, the 2DEG depletes. In Fig. 1(b), right, we show a simulation for $d=75 \mathrm{~nm}$, i.e., when all layers down to the $n$-GaAs are etched away. The 2DEG is then the only populated electron system between the contacts. Magnetotransport data, taken on a corresponding Hall bar, display clear Shubnikov-de Haas oscillations [Fig. 1(c)], characteristic for a high-quality 2DEG without parallel conductance. From magnetotransport we get an electron density $n=2.3 \times 10^{11} \mathrm{~cm}^{-2}$ and a mobility $\mu=5 \times 10^{5} \mathrm{~cm}^{2} / \mathrm{V} \mathrm{s}$ at $T=1.6 \mathrm{~K}$, from which we extract $l_{\mathrm{mf}} \approx 4 \mu \mathrm{m}$ and the diffusion constant $D=4200 \mathrm{~cm}^{2} / \mathrm{s}$. The functionality of the Esaki diode is shown in Fig. 1(d): a kink in $I-V$ characteristics at positive bias, marked with an arrow, is typical for Esaki diodes and confirms efficient Esaki diode operation.

To study spin injection we used NLSV measurements. According to the standard (diffusive) model of spin injection [25-27], the nonlocal voltage $V_{\mathrm{NL}}$ [see Fig. 1(a)] is a measure of a spin accumulation and is given by

$$
V_{\mathrm{NL}}= \pm P_{\text {inj }} P_{\text {det }} I R_{\text {sheet }} \lambda_{\mathrm{sf}} / 2 w \times \exp \left(-L / \lambda_{s f}\right)
$$

for the injected charge current $I$. The \pm sign corresponds to parallel $(+)$ and antiparallel $(-)$ injector-detector magnetization configuration. $R_{\text {sheet }}, \lambda_{\mathrm{sf}}$, and $L$ are the sheet resistance, the spin diffusion length, and the injectordetector separation, respectively. $P_{\text {inj(det) }}$ is the spin injection (detection) efficiency, equal to the tunnel spin polarization of the Esaki diode [2].

The results of the NLSV measurements are summarized in Fig. 2. Figure 2(a) displays $V_{\mathrm{NL}}$ at contact 3 for $I_{21}=-15 \mu \mathrm{A}\left(V_{3 T} \sim-0.44 \mathrm{~V}\right)$ as a function of magnetic field, swept in the $x$ direction. A clear NLSV signal is observed with the amplitude $\Delta V_{\mathrm{NL}}$ decaying exponentially with $L$ [28]. Such exponential decay is consistent with the assumption of diffusive transport between the contacts but does not exclude nondiffusive transport beneath the contacts. Using Eq. (1). we obtained $\lambda_{\text {sf }} \sim 3.2 \mu \mathrm{m}$, which is much smaller than the maximal $L_{2-5}$. Taking $D=4200 \mathrm{~cm}^{2} / \mathrm{s}$, we calculated a spin relaxation time $\tau_{s}=\lambda_{\mathrm{sf}}^{2} / D=24 \mathrm{ps}$, which is in the range observed in other 2D systems [29]. Given this value, one does not expect to observe a depolarization signal in an out-of-plane magnetic field due to Hanle effect [30].

Whereas the spatial dependence of the spin signal is well described by Eq. (1), its magnitude is not. In Fig. 2(b) we plot (red circles) the evolution of $\Delta R_{\mathrm{NL}}=\Delta V_{\mathrm{NL}} / I$ with bias voltage for $I$ between -100 and $+100 \mu \mathrm{A}$. For comparison, we also plot (black line) the signal calculated using Eq. (1) and assuming that $P_{\text {inj }}$ behaves as in bulk spin Esaki diode devices [2,21]. There, $P_{\text {inj }}$ is largest at low bias (we used $P_{\text {inj }}=75 \%$ [23]) and drops monotonically with increasing bias voltage [31]. We note two striking features in the case 

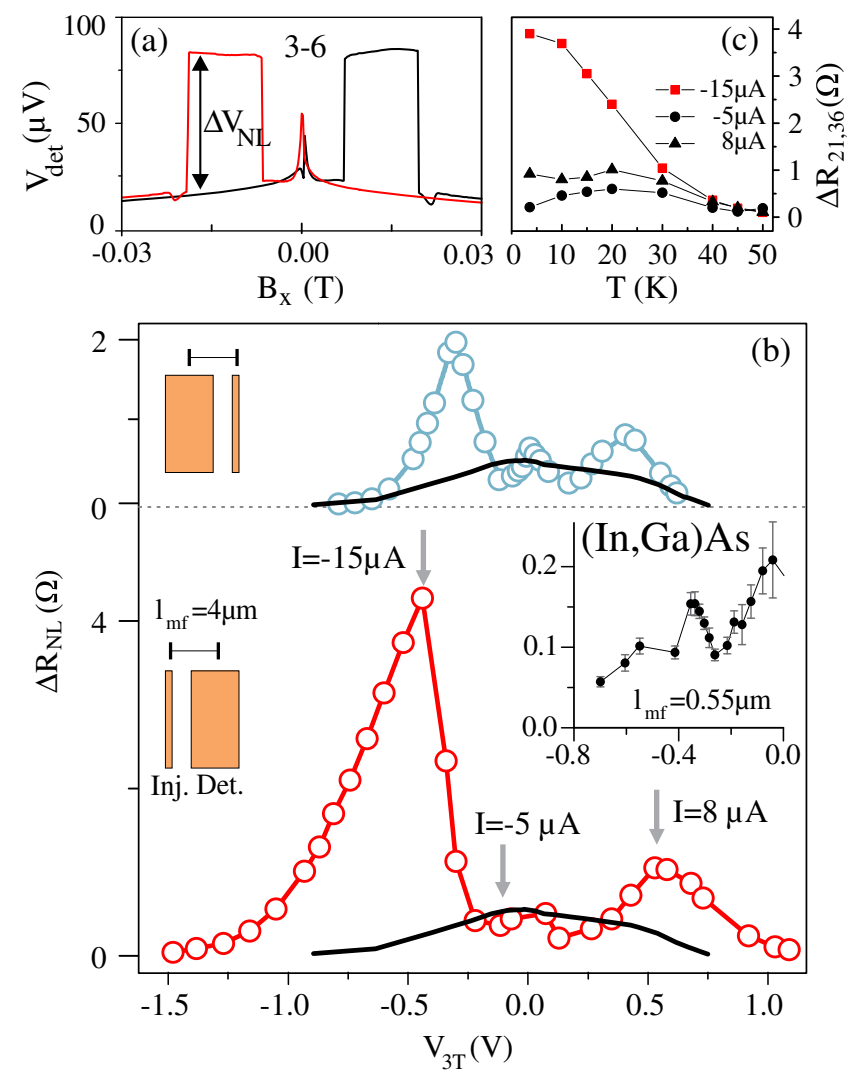

FIG. 2 (color online). (a) Nonlocal voltage at contact 3 for $I_{21}=-15 \mu \mathrm{A}$ versus in-plane magnetic field $B_{x}$ at $T=3.8 \mathrm{~K}$; field-dependent background has been removed from the raw data. (b) Bias dependence of the amplitude $\Delta R_{\mathrm{NL}}$ of the NLSV signal plotted versus $V_{3 T}$ for the narrow-wide (red circles) and widenarrow (blue) configurations, as defined in the text. Sketches on the left-hand side show how the width of injector and detector contacts in the each configuration compares to the mean free path $l_{\mathrm{mf}}$. Solid black lines correspond to the expected amplitude of the signal calculated using Eq. (1). We used $P_{\text {det }}=75 \%$ and $P_{\text {inj }}=$ $P_{\text {det }}$ at low bias. Inset: Bias dependence of the NLSV signal from a low mobility (In,Ga)As QW structure with $l_{\mathrm{mf}}=0.55 \mu \mathrm{m}$, for the narrow-wide configuration. (c) Temperature dependence of the NLSV signal's amplitude for the narrow-wide configuration for three injector current values, marked with arrows in (b).

of the 2D sample: (i) a nonmonotic behavior of the signal, never observed in our previous work on devices with 3D GaAs channels, and (ii) the amplitude of the signal for higher $\left|V_{3 T}\right|$ significantly exceeding values predicted by Eq. (1). For the negative-bias maximum, the measured signal is by a factor of $\sim 20$ larger. The enhanced spin signal clearly indicates shortcomings of the used spin drift-diffusion model. These deficiencies are particularly evident when one extracts $P_{\text {inj }}$ from the data using Eq. (1), with the biasindependent $P_{\text {det }}$ obtained from low-bias measurements where $P_{\text {det }}=P_{\text {inj }}$ holds [2]. For low-bias voltages, i.e., for $V_{3 T}$ between -250 and $+250 \mathrm{mV}$, we extract $P_{\text {inj }} \approx 75 \%$. For higher voltages, however, one gets highly unphysical values of $P_{\text {inj }}$ exceeding 100\%: 150\% and $\sim 650 \%$ for the positive- and the negative-bias peak, respectively.

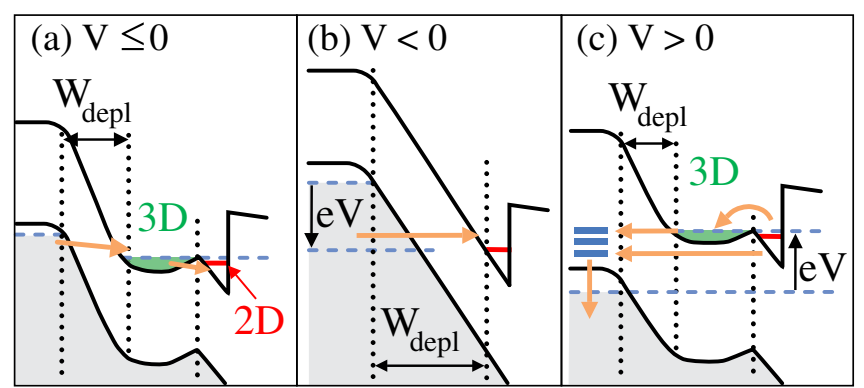

FIG. 3 (color online). Possible tunneling processes, indicated by orange arrows, at different bias. Shown is the region of intermediate bulk states (3D), 2DEG, and depletion width $w_{\text {depl }}$. (a) Low negative bias: spins are injected into $2 \mathrm{DEG}$ via $3 \mathrm{D}$ bulk states. (b) High negative bias: spins are injected directly into 2DEG (3D region depleted). (c) Excess current regime: spins are extracted from the 2DEG via localized states in (Ga,Mn)As band gap, both with and without the contribution of 3D states.

The fact that the experimental features discussed above were not observed in $3 \mathrm{D}$ devices strongly points to the presence of the $2 \mathrm{DEG}$ as their origin. As a possible key to an understanding of the experimental results, let us discuss how the applied bias affects electrical transport between $(\mathrm{Ga}, \mathrm{Mn})$ As and the 2DEG. Corresponding illustrations are shown in Fig. 3. Figure 3(a) shows the case of low negative bias $V \leq 0$. In this regime electrons tunneling between (Ga,Mn)As and the 2DEG have to travel via intermediate 3D states and are expected to generate a spin accumulation in the mildly doped $n$-GaAs [see also Fig. 1(b)]. The situation is then similar to the double-step tunneling discussed for the case where localized states at the FMsemiconductor interface play the role of the intermediate states [32,33]. If the coupling resistance between the $3 \mathrm{D}$ and 2D states were sufficiently large, then the spin accumulation in the 2DEG would be suppressed. Based on the simulation of the band profile of our structures [see Fig. 1(b)], we assume this coupling resistance to be low. This is consistent with the high spin injection efficiency $P_{\text {inj }}=75 \%$ of the injector contact extracted for this low-bias regime.

The band bending under forward bias, where we observe a peak in the NL signal, is shown in Fig. 3(c). There the current through the Esaki diode is dominated by the excess current [34], a result of multistep tunneling through impurity-related band gap states in the highly disordered $(\mathrm{Ga}, \mathrm{Mn})$ As. Direct tunneling between the $(\mathrm{Ga}, \mathrm{Mn}) \mathrm{As}$ VB and the $n$-GaAs conduction band (CB), dominating for negative and low positive bias, is suppressed as the overlap of the bands is lifted. It was demonstrated in experiments on bulk samples that tunneling between 3D electron states and the gap states does not enhance the NLSV signal in bulk channels [35]. We can assume that this also holds here and that this transport mechanism, marked by the upper arrow in Fig. 3(c), does not enhance the signal. There exists, however, an additional possible transport 
mechanism, i.e., resonant tunneling between impurityrelated states in the band gap and 2D states in the channel [lower arrow in Fig. 3(c)]. One can expect that this transport mechanism, absent in 3D structures, is causing the signal enhancement in a similar way as in the case of large negative bias (see below).

The situation for junctions under large reverse bias, for which we observe the largest enhancement, is depicted in Fig. 3(b). The large negative voltage increases the diode's depletion width $w_{\text {depl }}$. At a certain voltage one can expect that the 3D $n$-GaAs system will become completely depleted. Therefore, in contrast to the low-negative and also the positive-bias case, there will be no 3D states available for tunneling. As a result, electrons can now be injected from the $(\mathrm{Ga}, \mathrm{Mn}) \mathrm{As}$ directly into the 2D region, as depicted in Fig. 3(b). In summary, we conclude that in the low-bias region [Fig. 3(a)] the injection path involves 3D states, while at large negative bias [Fig. 3(b)] direct tunneling occurs between $(\mathrm{Ga}, \mathrm{Mn}) \mathrm{As}$ and the 2DEG. At positive bias [Fig. 3(c)], tunneling through midgap states in $(\mathrm{Ga}, \mathrm{Mn}) \mathrm{As}$ occurs, both with and without 3D states involved.

We note that the increase of the signal at large bias cannot be simply assigned to an increased $P_{\text {inj }}$, as the values extracted using Eq. (1) give $\sim 650 \%$ for the negative-bias peak and $\sim 150 \%$ for the positive-bias peak. Rather, we speculate that the enhancement is connected with $l_{\mathrm{mf}}$ being larger than the contact width. Ballistic motion underneath the injector contact might modify the conventional picture of spin injection and cause an enhanced spin current detected in NL measurements. Below we present experiments which support this picture although a microscopic model is not yet available.

The first of these experiments involved swapping injector and detector contacts. The measurements discussed so far were performed in a narrow-wide configuration, i.e., using the $0.5-\mu \mathrm{m}$-wide injector 2 and the $4-\mu \mathrm{m}$-wide detector 3. In Fig. 2(c) we also show the bias dependence of $\Delta R_{\mathrm{NL}}$ obtained for the wide-narrow configuration with the 4- $\mu \mathrm{m}$-wide contact 3 as the injector and contact 2 as detector (blue dots). Qualitatively, both curves are very similar [36], but the signals' amplitudes change, particularly at the reverse-bias maximum. With $\Delta R_{\mathrm{NL}} \approx 4.3 \Omega$, it is stronger for the former configuration, when the injector width is much smaller than $l_{\mathrm{mf}}$. For the latter one, it is $\approx 2 \Omega$. The signal at the forward-bias maximum follows this trend and is $\approx 1.05$ and $0.85 \Omega$, respectively. Although one cannot exclude current-related effects (the same voltage means higher current in case of a larger contact), one explanation for a correlation of the signal with the size of the contacts could involve ballistic effects [16].

Further correlation of the signal with $l_{\mathrm{mf}}$ is seen in the temperature evolution of $\Delta R_{\mathrm{NL}}$ for $T=3.8-50 \mathrm{~K}$, shown in Fig. 2(c). In this range, $l_{\mathrm{mf}}$ decreases from $\approx 4 \mu \mathrm{m}$ at $3.8 \mathrm{~K}$ to $\approx 2.4 \mu \mathrm{m}$ at $30 \mathrm{~K}$ and further to $\approx 1 \mu \mathrm{m}$ at $50 \mathrm{~K}$
[28]. In Fig. 2(c) we plot $\Delta R_{\mathrm{NL}}(T)$ measured in the narrowwide configuration for three bias values, marked with arrows in Fig. 2(b): $I=-15,-5$, and $+8 \mu \mathrm{A}$. Whereas the amplitude of the signal does not change much for $I=-5$ and $+8 \mu \mathrm{A}$ up to $T=30 \mathrm{~K}$, it drops very fast for $I=-15 \mu \mathrm{A} . \Delta R_{\mathrm{NL}}(T)$ at the negative-bias peak follows closely $l_{\mathrm{mf}}(T)$, suggesting that ballistic effects are particularly important in this bias regime, i.e., when no 3D bulk states are involved. At $T=50 \mathrm{~K}$, with $l_{\mathrm{mf}}$ only slightly larger than the width of contact 2, the enhancement of $\Delta R_{\mathrm{NL}}$ is gone and the bias dependence resembles the one observed for bulk devices, showing a decrease of $\Delta R_{\mathrm{NL}}$ with increasing $\left|V_{3 T}\right|$ [black lines in Fig. 2(b)].

Finally, a correlation between $l_{\mathrm{mf}}$ and the NLSV signal becomes evident by comparing the presented results with the ones obtained from similar measurements on a 2DEG in an (In,Ga)As quantum well. The latter has a mean free path $l_{\mathrm{mf}} \approx 0.55 \mu \mathrm{m}$, about 7 times smaller than in the (Al,Ga)As system. $\Delta R_{\mathrm{NL}}$ obtained in the narrow-wide configuration from this device is plotted in the inset of Fig. 2(b). Like in bulk devices, the signal is the strongest at low bias, and drops with increasing voltage, but the remainder of the reverse bias maximum survives at $V_{3 T}=-0.34 \mathrm{~V}$.

Last, let us briefly discuss results obtained from Hanle experiments. The measurements performed at low bias gave results consistent with $\tau_{s}=24$ ps; i.e., no depolarization curve was observed $[28,30]$. Consistent with that, we observe no dynamic nuclear depolarization (DNP) peak at NLSV traces at $B=0$, always observed for spin injection in bulk GaAs and stemming from hyperfine interaction with nuclear spins [37,38]. However, at negative bias, when $\Delta V_{\mathrm{NL}}$ becomes maximum, both a Hanle type of signal and the DNP peak appear (see Fig. 2(a) and [28]). The narrow Hanle-like curve corresponds to $\tau_{s} \approx 5 \mathrm{~ns}$. The reason for the narrow Hanle curve is most likely DNP in the 2D channel appearing only when $\Delta V_{\mathrm{NL}}$, and thus the spin current through the 2DEG is sufficiently high.

To summarize, we observed a clear spin valve signal for spin injection into a high mobility 2DEG at an $(\mathrm{Al}, \mathrm{Ga}) \mathrm{As} / \mathrm{GaAs}$ interface. The amplitude of the signal is strongly dependent on bias and can be tuned to values much larger than expected from the standard, diffusive model of spin injection. This, together with the observed correlation of the signal with the $l_{\mathrm{mf}}$ of the channel, suggests that ballistic effects need to be taken into account. To guide further experiments on spin injection into high mobility 2DEGs, a ballistic theory of spin injection is urgently needed.

This work has been supported by Deutsche Forschungsgemeinschaft (DFG) through SFB689.

*mariusz.ciorga@ur.de

[1] X. Lou, C. Adelmann, S. A. Crooker, E. S. Garlid, J. Zhang, K. S. M. Reddy, S. D. Flexner, C. J. Palmström, and P. A. Crowell, Nat. Phys. 3, 197 (2007). 
[2] M. Ciorga, A. Einwanger, U. Wurstbauer, D. Schuh, W. Wegscheider, and D. Weiss, Phys. Rev. B 79, 165321 (2009).

[3] S. P. Dash, S. Sharma, R. S. Patel, M. P. de Jong, and R. Jansen, Nature (London) 462, 491 (2009).

[4] R. Jansen, Nat. Mater. 11, 400 (2012).

[5] S. Datta and B. Das, Appl. Phys. Lett. 56, 665 (1990).

[6] N. Tombros, J. Csaba, M. Popinciuc, H. T. Jonkman, and B. J. van Wees, Nature (London) 448, 571 (2007).

[7] B. Birkner, D. Pachniowski, A. Sandner, M. Ostler, T. Seyller, J. Fabian, M. Ciorga, D. Weiss, and J. Eroms, Phys. Rev. B 87, 081405 (2013).

[8] A. T. Filip, B. H. Hoving, F. J. Jedema, B. J. van Wees, B. Dutta, and S. Borghs, Phys. Rev. B 62, 9996 (2000).

[9] P. R. Hammar and M. Johnson, Phys. Rev. Lett. 88, 066806 (2002).

[10] H. C. Koo, J. H. Kwon, J. Eom, J. Chang, and M. Johnson, Science 325, 1515 (2009).

[11] R. Jansen, B.-C. Min, and S. P. Dash, Nat. Mater. 9, 133 (2010).

[12] H. X. Tang, F. G. Monzon, R. Lifshitz, M. C. Cross, and M. L. Roukes, Phys. Rev. B 61, 4437 (2000).

[13] C.-M. Hu and T. Matsuyama, Phys. Rev. Lett. 87, 066803 (2001).

[14] V. Y. Kravchenko and E. I. Rashba, Phys. Rev. B 67, 121310(R) (2003).

[15] J.-S. Jeong and H.-W. Lee, Phys. Rev. B 74, 195311 (2006).

[16] A. Fert, J. George, H. Jaffrès, and R. Mattana, IEEE Trans. Electron Devices 54, 921 (2007).

[17] S. B. Kumar, M. B. A. Jalil, and S. G. Tan, Phys. Rev. B 75, 155309 (2007).

[18] A. Einwanger, M. Ciorga, U. Wurstbauer, D. Schuh, W. Wegscheider, and D. Weiss, Appl. Phys. Lett. 95, 152101 (2009).

[19] M. Kohda, Y. Ohno, K. Takamura, F. Matsukura, and H. Ohno, Jpn. J. Appl. Phys. 40, L1274 (2001).

[20] E. Johnston-Halperin, D. Lofgreen, R. K. Kawakami, D. K. Young, L. Coldren, A. C. Gossard, and D. D. Awschalom, Phys. Rev. B 65, 041306 (2002).

[21] P. V. Dorpe, Z. Liu, W. V. Roy, V. F. Motsnyi, M. Sawicki, G. Borghs, and J. D. Boeck, Appl. Phys. Lett. 84, 3495 (2004).
[22] M. Johnson and R. H. Silsbee, Phys. Rev. Lett. 55, 1790 (1985).

[23] M. Ciorga, M. Utz, D. Schuh, D. Bougeard, and D. Weiss, Phys. Rev. B 88, 155308 (2013).

[24] A. Trellakis, T. Zibold, T. Andlauer, S. Birner, R. Smith, R. Morschl, and P. Vogl, J. Comput. Electron. 5, 285 (2006).

[25] A. Fert and H. Jaffrès, Phys. Rev. B 64, 184420 (2001).

[26] E. Rashba, Eur. Phys. J. B 29, 513 (2002).

[27] J. Fabian, A. Matos-Abiague, C. Ertler, P. Stano, and I. Žutić, Acta Phys. Slovaca 57, 565 (2007).

[28] See Supplemental Material at http://link.aps.org/ supplemental/10.1103/PhysRevLett.113.236602 for further details.

[29] D. Stich, J. Zhou, T. Korn, R. Schulz, D. Schuh, W. Wegscheider, M. W. Wu, and C. Schüller, Phys. Rev. Lett. 98, 176401 (2007).

[30] For $\tau_{s}=\lambda_{\mathrm{sf}}^{2} / D=24 \mathrm{ps}$ one needs the field of $\sim 1 \mathrm{~T}$ to observe a depolarization curve. Therefore, it is difficult to perform Hanle measuremenst on such a system, especially given that the magnetization of the $(\mathrm{Ga}, \mathrm{Mn}) \mathrm{As}$ layer in our samples, i.e., orientation of injected spins, rotates out of plane between $B=0.2$ and $0.3 \mathrm{~T}$.

[31] We used values of $P_{\text {inj }}\left(V_{3 T}\right)$ as extracted from experiments on a bulk sample.

[32] M. Tran, H. Jaffrès, C. Deranlot, J.-M. George, A. Fert, A. Miard, and A. Lemaître, Phys. Rev. Lett. 102, 036601 (2009).

[33] R. Jansen, A. M. Deac, H. Saito, and S. Yuasa, Phys. Rev. B 85, 134420 (2012).

[34] A. G. Chynoweth, W. L. Feldmann, and R. A. Logan, Phys. Rev. 121, 684 (1961).

[35] J. Shiogai, M. Ciorga, M. Utz, D. Schuh, M. Kohda, D. Bougeard, T. Nojima, J. Nitta, and D. Weiss, Phys. Rev. B 89, 081307 (2014).

[36] The maxima for both configurations are observed at slightly different $V_{3 T}$. The corresponding shift is consistent with the one observed in $I-V$ curves shown in Fig. 1(d), where the characteristic kink is also observed at different $V_{3 T}$ for both used injector contacts.

[37] G. Salis, A. Fuhrer, and S. F. Alvarado, Phys. Rev. B 80, 115332 (2009).

[38] M. K. Chan, Q. O. Hu, J. Zhang, T. Kondo, C. J. Palmstrøm, and P. A. Crowell, Phys. Rev. B 80, 161206 (2009). 